\title{
Not just noise: Individual differences in general startle reactivity predict startle response to uncertain and certain threat
}

\author{
Daniel E. Bradford, Jesse T. Kaye, and John J. Curtin \\ Department of Psychology, University of Wisconsin-Madison, WI, USA
}

\begin{abstract}
General startle reactivity reflects defensive reactivity independent of affective foreground. We examined the relationship between General startle reactivity and startle response to threat in 3 tasks with distinct manipulations of threat uncertainty. General startle reactivity was a stronger predictor of startle response during threat (vs. no threat) and uncertain (vs. certain threat). These results confirm that including General startle reactivity in our analyses can increase the power and/ or precision to test effects of other focal experimental manipulations or grouping variables. Moreover, this suggests that individual differences in defensive reactivity moderate responding to threats of various types in our environment. As such, individual differences in General startle reactivity may index important psychological attributes related to trait affectivity, premorbid vulnerability for psychopathology, and manifest psychopathology.
\end{abstract}

Individual differences in defensive reactivity may index important psychological attributes related to trait affectivity, premorbid vulnerability for psychopathology, and manifest psychopathology (Vaidyanathan, Patrick, \& Cuthbert, 2009; Grillon \& Baas 2003). Substantial research has focused on the modulation of the startle response, a defensive reflex, by foreground affective stimuli in laboratory tasks. For example, the startle response is potentiated in the presence of a conditioned stimulus that has been paired with electric shock ("fear-potentiated startle"). Similarly, the startle response is increased when viewing unpleasant pictures and decreased during pleasant pictures ("valence-modulated startle"). These modulations of the startle response have been valuable tools in affective science. However, defensive reactivity may also be measured independent of foreground affective stimuli. Vaidyanathan et al. (2009) specified that general startle reactivity "refers to average startle reactivity in the absence of or without regard to foreground stimulus manipulations, if present" (p. 911). Although less well-studied than task startle modulation, measurement of General startle reactivity may yield important methodological and theoretical benefits.

From the methodological vantage point, including General startle reactivity as an additional independent variable in our analytic models may increase our power to test the effects of other focal experimental manipulations and the precision to estimate the magnitude of these other effects. This is particularly true for within-subject and between-subject experimental manipulations because these manipulations will be essentially uncorrelated with individual differences in General startle reactivity (Miller \& Chapman, 2001).

More importantly, General startle reactivity may also serve as a neurobiological indicator of dispositional defensive reactivity (Vaidyanathan et al., 2009). As such, General startle reactivity may identify individuals who will display exaggerated responding to affective stimuli or more potent effects of drugs and/or drug deprivation. It could also account for

Corresponding Author: John J. Curtin Department of Psychology University of Wisconsin - Madison 1202 West Johnson St., Madison, WI, 53706 Phone: 608-262-0387 jjcurtin@wisc.edu. 
heterogeneity within patient groups in clinical studies or mark premorbid risk for psychopathology. This suggests that General startle reactivity may interact with other meaningful experimental manipulations (e.g., shock threat, picture valence) or grouping variables (e.g., psychopathology status, drug vs. no-drug groups) to predict startle response. Identification of such interactions can clarify the relationships between these neurobiological processes, psychological traits, and psychopathology. Of course, modeling these interactions in our analyses, when significant, will further increase our statistical power.

In this brief report, we examine the utility of measuring General startle reactivity in tasks that test the effect of certain vs. uncertain threat on startle potentiation. Grillon and colleagues have demonstrated that startle response during uncertain (e.g., unpredictable) vs. certain (e.g., predictable) threat can distinguish patients with anxiety disorders from healthy controls (Davis, Walker, Miles, \& Grillon, 2010). Startle response during uncertain vs. certain threat is also sensitive to the acute administration and/or deprivation of various drugs (e.g., alcohol, benzodiazepines, nicotine, marijuana; (Davis et al., 2010; Gloria, 2011; Hogle, Kaye \& Curtin, 2010; Moberg \& Curtin, 2009). We tested three hypotheses in archival data from three distinct threat uncertainty tasks: 1) General startle reactivity will positively predict overall startle response, 2) the General startle reactivity - task startle response relationship will be stronger during threat than no-threat, and 3) the General startle reactivity - task startle response relationship will be stronger during uncertain than certain threat. Confirmation of these hypotheses would provide preliminary evidence that General startle reactivity may be an important affect and psychopathology-relevant individual difference marker. Furthermore, this would indicate that including General startle reactivity in our analytic models can increase power/precision to test effects of our experimental manipulations.

\section{Method}

\section{Participants}

Participants were originally recruited from the university and surrounding community for six separate experiments that examined the effects of alcohol administration or drug (i.e., nicotine or marijuana) deprivation on startle response during certain and uncertain threat of shock. The final sample consists of 348 participants (160 female), ages 18-50 ( $\mathrm{M}=23.7$; $\mathrm{SD}=5.4)^{1}$ from the "control" groups of these experiments who completed the threat task only once. Therefore, none of these participants were administered alcohol or in acute withdrawal following extended deprivation from nicotine or marijuana. The sample included 170 recreational, non-problematic users of alcohol who were in the "sober" control group of four alcohol administration experiments; 16 non-smokers and 26 non-deprived smokers were included from the two control groups of one nicotine deprivation experiment; 73 nonmarijuana users and 63 non-deprived marijuana users were included from the two control groups of one marijuana deprivation experiment.

\section{General Startle Reactivity and Shock Tolerance Assessments}

In all tasks, General startle reactivity was measured to acoustic probes during a baseline procedure that included a series of brief colored square "cues" presented on a CRT monitor. Cues were matched in size, shape, and duration to those presented during the respective main tasks described below. Acoustic probes were presented during both cues and the intertrial intervals (ITI) between cues. No shocks were administered during this procedure.

\footnotetext{
${ }^{1}$ We removed four additional participants that were identified as regression outliers (i.e., studentized residual with Bonferroni corrected $\mathrm{p}<0.05$ ) in preliminary GLM analyses.
} 
Following this procedure, shock electrodes were attached and participants' maximum shock tolerance was measured via standard procedures in our laboratory (Hefner \& Curtin, 2012).

\section{Threat Uncertainty Tasks}

Detailed methods for the three tasks have been published previously (see citations for each task below). Each of the tasks included fully counterbalanced No shock, Certain shock, and Uncertain shock conditions. In all conditions, participants were presented with colored square cues on a CRT monitor with a variable ITI. A message was presented on the monitor to indicate the condition for each upcoming block of trials.

The No-shock condition was comparable in all three tasks. Participants were instructed that no shocks would occur at any point during the cue or ITIs in this condition. The Certain shock condition was comparable across all three tasks as well. Participants were instructed that shocks were completely predictable with shocks administrated during every cue (100\% probable) at a known time (end of brief cues) in this condition. This resulted in 10, 15, and 10 total shocks across Certain shock conditions for the No-Shock/Predictable Shock/ Unpredictable Shock (NPU), Probability, and Duration tasks, respectively.

Shock contingencies in the Uncertain shock condition differed across the three tasks. In the uncertain shock condition of the NPU task (Moberg \& Curtin, 2009; also see Schmitz \& Grillon, 2012), shocks were administered unpredictably and could occur at any point during the cues or ITIs (5 shocks total). In the Probability task (Hefner \& Curtin, 2012), shocks were administered at a known time (end of brief cues) but only during $20 \%$ of the cues in the uncertain condition (5 shocks total). This introduced uncertainty regarding which cues would be shocked. In the Duration task (Hefner, Moberg, Hachiya, \& Curtin, 2013), shocks were administered at the end of all cues (100\% probable; 12 shocks total) but participants were instructed that cues could vary in duration from 5 seconds to 3 minutes in the uncertain condition. This introduced uncertainty regarding the timing of the shocks.

\section{Startle Measurement}

We sampled electromyographic activity (2000 Hz sampling rate with $500 \mathrm{~Hz}$ low-pass filter) in the orbicularis oculi muscle from electrodes under the right eye (Blumenthal et al., 2005). We measured eyeblink startle response to white noise acoustic probes (50 ms, $102 \mathrm{~dB}$ ) presented during the baseline and main tasks. We balanced the serial position of the probes across conditions within subjects during the three tasks.

Startle response processing included high-pass filtering (4th order $28 \mathrm{~Hz}$ Butterworth filter), signal epoching (-50-250 ms surrounding noise probe), rectification, and smoothing (4th order $30 \mathrm{~Hz}$ Butterworth low-pass filter). Trials with greater than $40 \mu \mathrm{V}$ deflections in the $50 \mathrm{~ms}$ pre-probe baseline were rejected as artifact. We scored peak response between 20 and $120 \mathrm{~ms}$ post-probe onset relative to mean $50 \mathrm{~ms}$ pre-probe baseline. Participants with $\geq 20 \%$ of trials where the response amplitude in the scoring window did not exceed the maximum pre-probe amplitude (non-responders) and/or $\geq 20 \%$ excessive artifact trials were excluded from analysis ${ }^{2}$. We calculated General startle reactivity as the mean response to all probes presented during the baseline procedure.

\section{Results}

We analyzed startle response during the three threat tasks in a General Linear Model (GLM) with repeated measures for Condition (No shock, Uncertain shock, Certain shock). We

\footnotetext{
${ }^{2}$ We removed an additional 16 non-responders and 6 participants with excessive artifact during data processing.
} 
included fully interactive between-subject regressors for General startle reactivity (meancentered) and Task (NPU, Probability, Duration). Condition effects were parsed into two orthogonal contrasts: (1) Overall threat: Average of uncertain shock and certain shock versus no shock, (2) Uncertain threat: Uncertain shock vs. certain shock. The interactions between General startle reactivity and these orthogonal contrasts allowed us to statistically compare the magnitude of General startle reactivity's influence on startle response across No shock, Uncertain shock, and Certain shock conditions. We report both raw GLM coefficients (b) and partial eta-squared $\left(\eta_{\mathrm{p}}{ }^{2}\right)$ to describe effect sizes.

As expected, the Overall threat contrast was significant, $b=50.14, t(342)=21.21, p<0.001$, $\eta_{\mathrm{p}}{ }^{2}=0.57$, indicating that task startle response increased during both shock conditions relative to the no shock condition. The Uncertain threat contrast was also significant, $\mathrm{b}=7.71, t(342)=3.40, p<0.001, \eta_{\mathrm{p}}{ }^{2}=0.03$, indicating that task startle response increased during uncertain relative to certain shock.

The effect of General startle reactivity was significant such that startle response across tasks and conditions increased $0.78 \mu \mathrm{V}$ for every $1 \mu \mathrm{V}$ increase in General startle reactivity, $\mathrm{b}=0.78, t(342)=36.40, p<0.001, \eta_{\mathrm{p}}{ }^{2}=0.79$. The General startle reactivity $\mathrm{X}$ Overall threat contrast was significant, $\mathrm{b}=0.15, t(342)=5.96, p<0.001, \eta_{\mathrm{p}}{ }^{2}=0.09$, such that the relationship between General startle reactivity and task startle response was stronger during the two shock conditions (average $b=0.83$ ) than the no shock condition ( $b=0.68$ ). More specifically, this indicates that for every $1 \mu \mathrm{V}$ increase in General startle reactivity, task startle response increased by $0.83 \mu \mathrm{V}$ during the average of the two shock conditions, but only by $0.68 \mu \mathrm{V}$ during the no shock condition and this 0.15 difference in the magnitude of the two simple effects of General startle reactivity was significant.

The General startle reactivity $\mathrm{X}$ Uncertain threat contrast was also significant, $\mathrm{b}=0.10$, $t(342)=4.11, p<0.001, \eta_{\mathrm{p}}{ }^{2}=0.05$, such that the relationship between General startle reactivity and task startle response was stronger during uncertain $(\mathrm{b}=0.88)$ than certain shock $(\mathrm{b}=0.78)$. More specifically, this indicates that for every $1 \mu \mathrm{V}$ increase in General startle reactivity, task startle response increased by $0.88 \mu \mathrm{V}$ during uncertain shock, but only by $0.78 \mu \mathrm{V}$ during certain shock and this 0.10 difference in the magnitude of the two simple effects of General startle reactivity was significant. Figure 1A displays the relationship between task startle response and General startle reactivity as a function of Condition. Task did not moderate the effect of General startle reactivity or its interactions with Overall threat and Uncertain threat contrasts, indicating that these significant effects were comparable across the three tasks (see Figures 1B-D).

\section{Discussion}

We demonstrated that General startle reactivity was a robust predictor of task startle response overall and that it displayed stronger relationships with startle response during threat, and most specifically, uncertain threat. Moreover, we observed these relationships consistently across three threat tasks with distinct manipulations of uncertain threat, which increases confidence that this is a generalizable effect. Partial eta ${ }^{2}$ effect sizes indicated that including each of these effects of General startle reactivity reduced previously unexplained error variance for our tests of the effects of threat condition by between $10-15 \%$. This suggests that including General startle reactivity as an individual difference variable in our analyses of threat tasks can increase the power/precision with which we test the effects of other focal between- and within-subjects experimental manipulations (e.g., affect manipulations, drug administration/deprivation, randomized clinical trials) that will be uncorrelated with General startle reactivity. In experiments where power is lower due to relatively small effect sizes, $\mathrm{N}$, or other reasons (e.g., between-subject or mixed-model 
effects), the reduction in model error associated with including General startle reactivity in our analytic models may make the difference between detecting vs. failing to detect meaningful effects.

The significant interactions between General startle reactivity and overall threat and uncertain threat contrasts may also have theoretical importance. These interactions indicate that General startle reactivity measured during a putatively neutral baseline task is more tightly coupled with task startle responding during conditions of threat and in particular, uncertain threat, relative to periods of safety. By definition, General startle reactivity reflects the strength of trait reflexive defensive responding to a mildly aversive unconditioned stimulus (i.e., abrupt, loud noise). Our results suggest that individuals who display high trait defensive responding to unconditioned stimuli also display relatively stronger phasic modulation of this same reflexive defensive system by cues that predict impending environmental threats. Moreover, this coupling is strongest for uncertain threats. This latter observation has theoretical import because reactions to unpredictable or otherwise uncertain threats may represent a dimensional marker of the manifestation of anxious psychopathology including Post-Traumatic Stress Disorder and Panic Disorder (Davis et al., 2010). As such, General startle reactivity may contribute as a neurobiological index of fear circuitry from the emerging NIMH Research Domain Criteria perspective. Furthermore, it may be more easily measured than startle response potentiation to uncertain shock threat in populations for which shock is proscribed (e.g., children) or for laboratories who do not have the equipment or expertise to administer shock. Future research should examine if General startle reactivity can be measured comparably with response to probes during the ITIs or across all probes in our primary tasks rather than baseline procedures (see Vaidyanathan et al., 2009). If true, this individual difference may be easily indexed in all research that measures the startle response.

Future research should also examine the characteristics of baseline tasks that are necessary to observe the relationships involving General startle reactivity that we observed here. In the experiments reported here, measurement of General startle reactivity was obtained in an unfamiliar laboratory environment during a baseline period that immediately preceded a shock threat task. As such, there may be important individual differences in the degree that participants were already experiencing uncertainty due to either the unfamiliar baseline procedure or the distal shock threat task that would follow. Future research should confirm if similar effects are observed in baseline tasks after the participant has been habituated to the laboratory and procedures and while not anticipating a subsequent aversive task.

In other research, we have observed General startle reactivity to explain potentially important individual differences in processes that are implicated in drug addiction etiology. For example, Bradford, Shapiro \& Curtin (2013) observed that individuals who displayed increased General startle reactivity when sober experienced greater anxiolytic effects of alcohol (i.e., reduced startle potentiation to uncertain threat) than did comparably intoxicated individuals with lower baseline General startle reactivity. Conversely, in separate experiments we have demonstrated that drug-deprived heavy daily tobacco and heavy daily marijuana smokers who exhibit increased General startle reactivity at baseline respond more aversively to uncertain threats than similarly drug-deprived users with lower baseline General startle reactivity (Gloria, 2011; Hogle et al., 2010). These preliminary findings suggest that General startle reactivity may serve as an individual difference marker for the propensity to experience negative reinforcement from alcohol and other drug use in stressful contexts. Contemporary addiction theorists suggest that this reinforcement may result from neuroadaptation in the brain's stress circuitry following chronic drug use (Koob \& Volkow, 2010). As such, individual differences in General startle reactivity may be a risk marker for potential plasticity of these stress circuits. 
It should be acknowledged that measurement artifact may have contributed to the differential relationship between General startle reactivity and task startle response during threat conditions. Individuals who startle more vigorously in general may also show greater increases in startle during threat if the neural inputs from threat processing circuits are nonadditive. Of course, it is somewhat a matter of perspective if this is considered artifact or instead informative about the organization and function of these neural circuits.

Furthermore, it should be noted that the power/precision benefits associated with analyzing General startle reactivity remain regardless of the nature of these relationships. Modeling this large source of otherwise error variance will increase analytic power/precision in either case.

Researchers should examine whether General startle reactivity predicts the magnitude of startle modulation by affective stimuli other than shock cues (e.g., unpleasant/pleasant pictures, emotional imagery). If it does, General startle reactivity may prove to be a useful and informative individual difference variable more broadly in affective science research that uses startle response as a dependent measure. The construct validity of General startle reactivity can be further increased by assessing its convergent and discriminant validity using other physiological, self-report, and behavioral measures during both baseline startle assessments and experimental task manipulations. Researchers should also model interactions between General startle reactivity and experimental manipulations, grouping variables, and novel pharmacologic, behavioral, and psychosocial interventions in affective and clinical science. Situating General startle reactivity in this more detailed nomological network including other constructs that we either measure or manipulate across levels of analysis will be necessary to define and clarify this potentially important psychobiological individual difference.

\section{Acknowledgments}

Funding was provided by grants to D.E.B from NIMH (T32 MH018931-21), to JTK from NSF (DGE-0718123) and to J.J.C from NIAAA (R01 AA15384) and NIDA (R01 DA033809).

\section{References}

Blumenthal T, Cuthbert B, Filion D, Hackley S, Lipp O, van Boxtel A. Committee report: Guidelines for human startle eyeblink electromyographic studies. Psychophysiology. 2005; 42(1):1-15. doi: 10.1111/j.1469-8986.2005.00271.x. [PubMed: 15720576]

Bradford D, Shapiro B, Curtin J. How bad could it be? Alcohol dampens stress responses to uncertain intensity threat. Psychological Science. 2013; 24(12):2541-2549. doi:10.1177/0956797613499923. [PubMed: 24145332]

Davis M, Walker D, Miles L, Grillon C. Phasic vs sustained fear in rats and humans: role of the extended amygdala in fear vs anxiety. Neuropsychopharmacology. 2010; 35(1):105-135. [PubMed: 19693004]

Gloria, R. Doctoral dissertation. University of Wisconsin-Madison; 2011. Uncovering a potential biological marker for marijuana withdrawal: Startle potentiation to threat..

Hefner K, Curtin J. Alcohol stress response dampening: Selective reduction of anxiety in the face of uncertain threat. Journal of Psychopharmacology. 2012; 26:232-244. doi: 10.1177/0269881111416691. [PubMed: 21937686]

Grillon C, Baas J. A review of the modulation of startle reflex by affective states and its application to psychiatry. Clinical Neurophysiology. 2003; 114:1557-1579. doi:10.1016/S1388-2457(03)00202-5. [PubMed: 12948786]

Hefner K, Moberg C, Hachiya L, Curtin J. Alcohol stress response dampening during imminent vs. distal, uncertain threat. Journal of Abnormal Psychology. 2013; 122:756-69. doi:10.1037/ a0033407. [PubMed: 24016014] 
Hogle J, Kaye J, Curtin J. Nicotine withdrawal increases threat-induced anxiety but not fear: Neuroadaptation in human addiction. Biological Psychiatry. 2010; 68:719-725. doi:10.1016/ j.biopsych.2010.06.003. [PubMed: 20673878]

Koob G, Volkow N. Neurocircuitry of addiction. Neuropsychopharmacology Reviews. 2010; 35:217238. doi:10.1038/npp.2009.110. [PubMed: 19710631]

Miller G, Chapman J. Misunderstanding analysis of covariance. Journal of Abnormal Psychology. 2001; 110(1):40-48. doi:10.1037/0021-843X.110.1.40. [PubMed: 11261398]

Moberg C, Curtin J. Alcohol selectively reduces anxiety but not fear: startle response during unpredictable versus predictable threat. Journal of Abnormal Psychology. 2009; 118(2):335-347. doi:10.1037/a0015636. [PubMed: 19413408]

Schmitz A, Grillon C. Assessing fear and anxiety in humans using the threat of predictable and unpredictable aversive events (the NPU-threat test). Nature Protocols. 2012; 7(3):527-532. doi: 10.1038/nprot.2012.001.

Vaidyanathan U, Patrick C, Cuthbert B. Linking Dimensional Models of Internalizing Psychopathology to Neurobiological Systems: Affect-Modulated Startle as an Indicator of Fear and Distress Disorders and Affiliated Traits. Psychological Bulletin. 2009; 135(6):909-942. doi: 10.1037/a0017222. [PubMed: 19883142] 

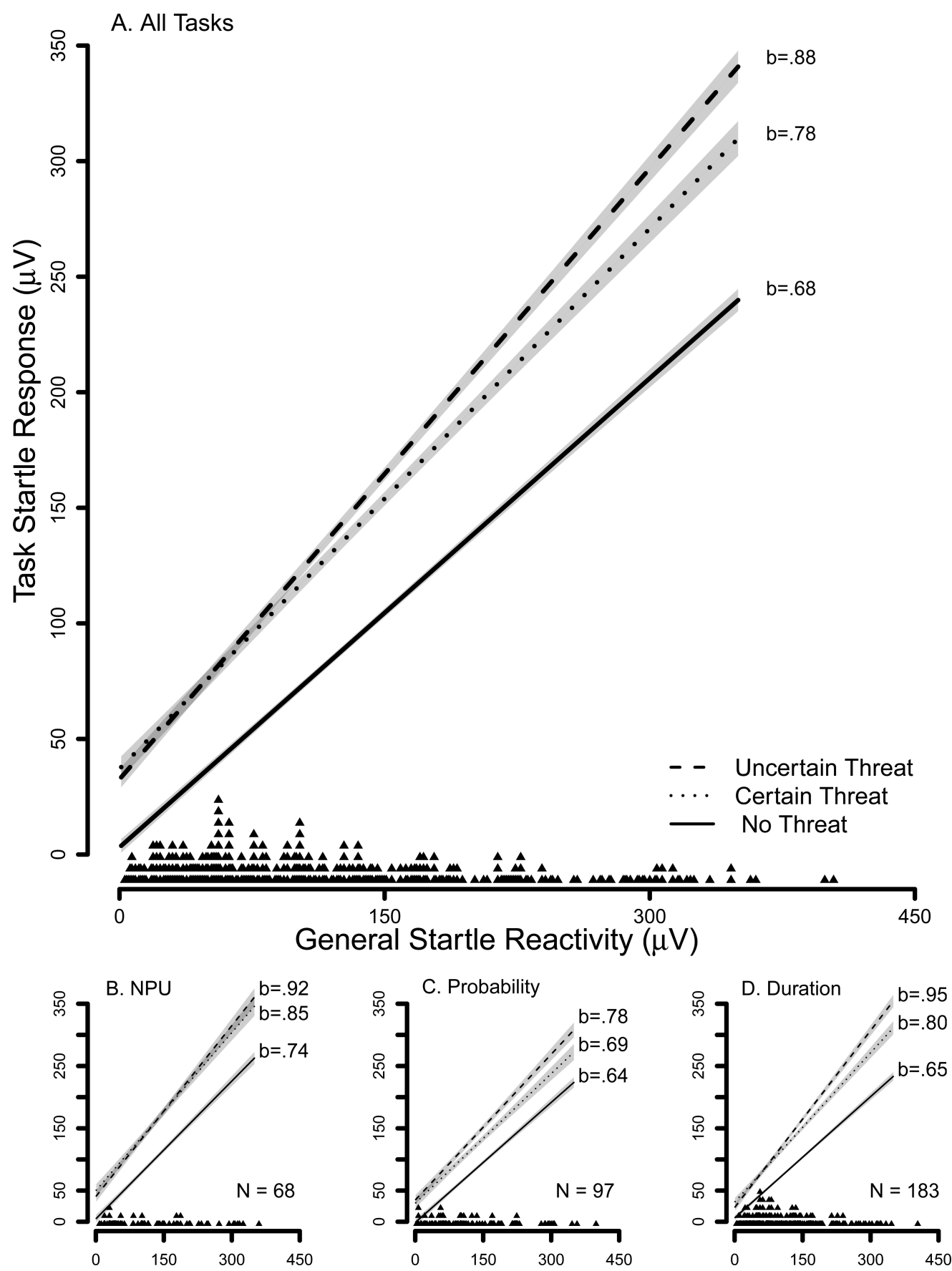

Figure 1. Startle Response by General Startle Reactivity and Condition

Gray bands indicate confidence envelopes (+/- one standard error) for point estimates of startle response from the general linear model (GLM). We included a strip plot of General startle reactivity for all participants along each x-axis. We report GLM coefficients for the simple effects in each condition. Panel A displays aggregate data across the three tasks. Panels B-D display data separately for the three tasks. 